\title{
System Model of Heat and Mass Transfer Process for Mobile Solvent Vapor Phase Drying Equipment
}

\author{
Shiwei Zhang, ${ }^{1}$ Yufang Zhu, ${ }^{1}$ Baozhen Qiao, ${ }^{2}$ and Zhijun Zhang ${ }^{1}$ \\ ${ }^{1}$ School of Mechanical Engineering and Automation, Northeastern University, Shenyang 110004, China \\ ${ }^{2}$ Shenyang Chengqiao Science and Technology Development Co., Ltd., Shenyang 110025, China \\ Correspondence should be addressed to Shiwei Zhang; shwzhang@mail.neu.edu.cn
}

Received 16 May 2014; Accepted 17 July 2014; Published 3 September 2014

Academic Editor: Jun Liu

Copyright (c) 2014 Shiwei Zhang et al. This is an open access article distributed under the Creative Commons Attribution License, which permits unrestricted use, distribution, and reproduction in any medium, provided the original work is properly cited.

\begin{abstract}
The solvent vapor phase drying process is one of the most important processes during the production and maintenance for large oilimmersed power transformer. In this paper, the working principle, system composition, and technological process of mobile solvent vapor phase drying (MVPD) equipment for transformer are introduced in detail. On the basis of necessary simplification and assumption for MVPD equipment and process, a heat and mass transfer mathematical model including 40 mathematical equations is established, which represents completely thermodynamics laws of phase change and transport process of solvent, water, and air in MVPD technological processes and describes in detail the quantitative relationship among important physical quantities such as temperature, pressure, and flux in key equipment units and process. Taking a practical field drying process of $500 \mathrm{KV} / 750 \mathrm{MVA}$ power transformer as an example, the simulation calculation of a complete technological process is carried out by programming with MATLAB software and some relation curves of key process parameters changing with time are obtained such as body temperature, tank pressure, and water yield. The change trend of theoretical simulation results is very consistent with the actual production record data which verifies the correctness of mathematical model established.
\end{abstract}

\section{Introduction}

Large power transformer is the important equipment of power transmission and transformation system. Insulation system of transformer is the key factor affecting the reliable operation of transformer. If the insulation system of transformer was affected with damp due to various reasons, its insulation performance and reliability would decrease. With the rapid development of power industry in China, the transformer drying process difficulty increases and higher quality requirements are required because of higher voltage class of transformer, larger capacity, and more insulating materials. Drying process during transformer manufacture occupies a very important position and it has always been a key link on affecting the transformer quality and production $[1,2]$.

Solvent vapor phase drying (VPD) process is one of the most important processes during the production and maintenance of large oil-immersed power transformer. Since it is the last process before oiling, it will directly affect the insulation class and voltage endurance of transformer. At present, because of its advantages in drying the large power transformer, the VPD equipment has become indispensable, ideal, reliable, and advanced equipment for drying the large power transformer with extrahigh voltage and large capacity $[3,4]$.

Due to transformer characteristics and actual demand, the power transformers are made larger and larger which brings much difficulty for transportation. Besides, large power stations are mostly built in the energy center at southwestern or northwestern area of China where the transportation condition is very poor. Therefore, the transportation cost in its entirety is expensive, and even entirety transportation is impossible sometimes. Thus, the ASA (advanced on site assemble) transformer emerges, and then the MVPD equipment emerges at the right moment for drying the ASA transformer.

The ASA transformer after the test can be directly divided into some small system components which can be conveniently transported. These small system components can be 
transported to the work place of power station and be assembled again. The transformer will inevitably be affected with damp during decomposition and assembly, so the VPD process for the transformer in the power station is necessary. Besides, The MVPD technology is also the most ideal choice for old transformer which needs maintenance on site.

In recent years, much attention has been paid to the MVPD equipment. Some companies in China have taken the lead in trial-producing several sets of MVPD equipment in the world which have been applied to deal with many sets of large transformer equipment. Multiple manufacturers are also developing and trial-producing the MVPD equipment in China $[5,6]$.

However, at present, related basic theory research about the MVPD technology is still weak. During the equipment manufacture and use, manufacturers and users mainly copy and imitate the actual experience data from stationary VPD devices for lacking theoretical guidance. However, many differences exist between the mobile VPD devices and original stationary VPD ones. For instance, distributed control and separate lines are adopted in the MVPD equipment which makes its structure more compact. Considering the transportation problem, every single component in the MVPD equipment system should not be too large. Transformer shell is directly used as vacuum chamber in the MVPD process. The MVPD equipment mainly uses electricity as original power while the stationary equipment uses coal-burning boiler as original power. These changes of MVPD equipment bring new problems to the designers, manufacturer, and users. They need to solve the problems combining with its specific characteristics.

In fact, there are few technical documents about MVPD technology at present, and most of them only give a qualitative introduction of MVPD processes. The heat and mass transfer for the MVPD processes and equipment are studied rarely, so the mechanism analysis is nearly blank. But research on this aspect has an important significance and function for the development of MVPD technology and devices [79]. For example, in the aspect of process control, judgment conditions for transformation from heating stage into depressurization stage and for ending the drying process are very important. If the judgment conditions can be predicted and controlled accurately by theoretical model, the drying efficiency will be greatly increased and the goal of saving energy and reducing consumption will be achieved [10-12].

In order to meet the practical needs of quantitative theoretical research and numerical simulation in process control, a comprehensive heat and mass transfer mathematical model for the MVPD process is set up in this paper. The quantitative relationship among the important physical quantities in the MVPD key device units and technical process are described in detail, such as temperature, pressure, and flux. The model may provide a theoretical reference for the manufacturers and users of MPVD equipment [8,13].

\section{Principle and Equipment Structure of MVPD Technology}

2.1. Working Principle of MVPD Equipment. According to the drying theory, the moisture in transformer insulation

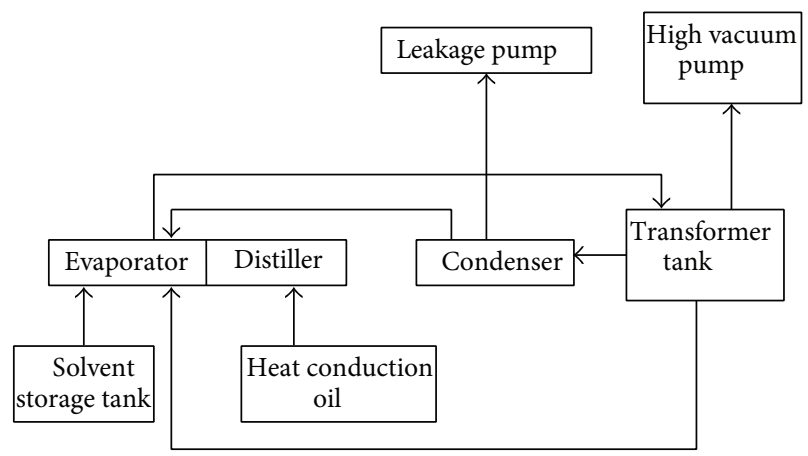

FIGURE 1: Structural schematic diagram of the MVPD system.

material mainly exists in the form of capillary adsorption. The drying process is that water molecules obtain enough energy to change into water vapor molecules and water vapor molecules diffuse into the surrounding space through capillary porosity of insulation material to break away from the insulation material. The motivation driving water vapor molecules to escape from insulation material is the pressure difference $\Delta P$ between the interior of material and the surrounding space. So the greater the $\Delta P$ is, the higher the drying rate is. There are mainly two ways to increase the pressure difference $\Delta P$. On the one hand, we can increase the temperature of insulation material so as to increase the saturated vapor pressure of its internal moisture. On the other hand, we can decrease the pressure of surrounding space [1].

The working principle of MVPD technology is as follows. The tank of power transformer is used as a vacuum chamber and is evacuated. The liquid solvent, a kind of kerosene oil, is adopted as the heat carrier and is vaporized into solvent vapor by an evaporator. The solvent vapor at high temperature enters vacuum chamber and condenses when it encounters the transformer body at low temperature. The phase change takes place and solvent vapor turns into liquid solvent again which will be sent into the evaporator and will be recycled. The temperature of whole transformer, including tank, iron core, copper wire, and insulation material, rises by absorbing the latent heat of phase changing from solvent vapor. The temperature of moisture inside the insulation material will also rise. And the internal moisture will escape from the insulation material because of the evaporation phase change. In order to increase the drying rate, vacuum process and heat process are alternated in turn to promote the condensation of solvent and the evaporation of moisture, until moisture content in insulation material meets the index requirements.

\subsection{System Structure of MVPD Equipment. The MVPD} equipment basically consists of the following systems: transformer tank, vacuum system, condensing system, solvent vapor system, solvent storage and delivery system, heat conduction oil heating system, pneumatic system, cooling water system, control system, and ventilation system. The principle block diagram is shown in Figure 1.

Modularized design pattern with double evaporators was adopted in the first set of the MVPD equipment made in China. Its system principle diagram is shown in Figure 2. This 


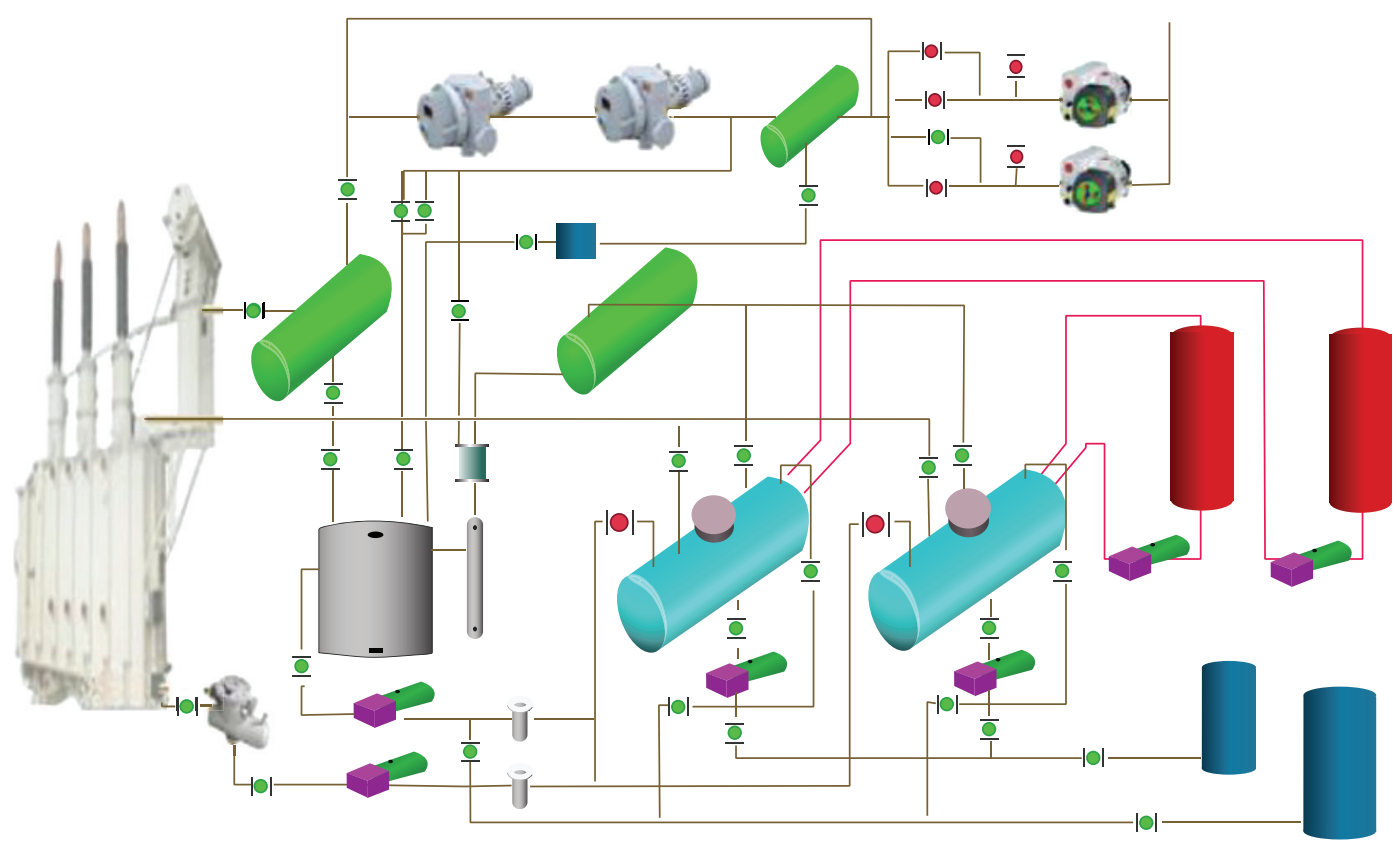

FIGURE 2: MVPD8.2 system principle diagram of MVPD equipment with two evaporators.

MVPD equipment is made up of the following cell modules [6]:

(a) main big module 1: vacuum unit and condensation module;

(b) main big module 2: evaporator and electric heater module;

(c) small equipment module: coarse filter and solvent circulating pump module;

(d) auxiliary equipment module 1: solvent tank and wasted oil tank module;

(e) auxiliary equipment module 2: water tank and water chiller unit, air compressor, and air tank;

(f) auxiliary component module: box loading connection pipes (two wide pipes and three thin tubes);

(g) thermal insulation blanket and tank heating device module.

2.3. Working Processes of MVPD Equipment. According to the time order, there are mainly the following processes when the MVPD equipment works $[1,14,15]$.

(1) Preparation Stage. At first, condensation system and evaporator are evacuated by the leakage pump. Then enough solvent liquid is injected into the evaporator from the solvent storage tank. The vacuum system is started and the transformer tank is evacuated until its pressure drops below $700 \mathrm{~Pa}$. In order to save time, the evaporator is preheated at the preparation stage and its temperature is controlled at $80^{\circ} \mathrm{C}$ to $90^{\circ} \mathrm{C}$.

(2) Heating Stage. Close the main vacuum valve and stop the vacuum system. Open the solvent vapor valve. Solvent in the evaporator is heated and changed into solvent vapor. Solvent vapor enters into the transformer tank, condenses on the cold surface of transformer body, and releases the latent heat of phase change. The condensed solvent liquid is discharged from the bottom of transformer tank and is then delivered back to the evaporator to reheat and vaporize. In this case, the transformer body is heated by the circular solvent vapor, so the body temperature is gradually getting higher. With the temperature increasing, moisture in insulation material starts to evaporate and diffuse gradually.

At the heating stage, the transformer tank is consistently evacuated by the leakage pump in order to ensure pressure difference between the transformer tank and the evaporator. The evacuated gas in transformer tank is the mixture including uncondensed solvent vapor, released water vapor, and leaking air. The mixed gas enters into the main condenser. Solvent vapor and water vapor condense into liquids and then flow into the collecting tank. Due to the different density, solvent and water are naturally separated by gravity settling. Water deposits at the bottom of collecting tank and is discharged while the solvent at upper layer is collected and delivered to evaporator, thus forming another cycle.

The temperature of solvent vapor should be controlled at $130^{\circ} \mathrm{C}$ to $135^{\circ} \mathrm{C}$ which is determined according to the maximum allowable temperature of class A insulation materials under the anaerobic condition. In the several times of heating stage, about $90 \%$ of the water content in insulation material may be drawn out.

(3) Intermediate Pressure Lower Stage. The condensation of solvent vapor will be restrained after the insulation material absorbing enough solvent, which will hinder both subsequent solvent vapor from condensation and internal moisture from migrating outwards. In order to increase the solvent condensation and the moisture migration, it is needed to stop heating stage and enter the intermediate pressure lower (IPL) stage. 
TABLE 1: Symbol definition in the simplified calculation process model of MVPD system.

\begin{tabular}{ll}
\hline Process units & Process logistics \\
\hline (A) Solvent storage tank & (1) Liquid solvent enters into the evaporator \\
(B) Evaporator & (2) Solvent vapor enters into transformer tank and starts to heat transformer \\
(C) Condenser & (3) Mixed gas enters into condensation system and condenses \\
(D) Transformer & (4) Leakage pump system evacuates the transformer tank through condensation system \\
(E) High vacuum system & (5) Solvent condensed by condensation system returns evaporator and is reused \\
(F) Leakage pump system & (6) Condensed liquid solvent flows into evaporator from the bottom of transformer tank and is reused \\
\hline
\end{tabular}

Close the solvent liquid inlet valve to stop solvent entering into the evaporator. Evacuate the transformer tank by the leakage pump so that the solvent remaining in the insulation material can evaporate again. Then a large amount of solvent vapor and partial water vapor enter into the main condenser, condense into liquids, and flow into the collecting tank. Separate the water and solvent in the collecting tank and make all solvent liquid back to the solvent storage tank. The intermediate pressure lower stage ends when the vacuum degree in the transformer tank reaches $4.5 \mathrm{kpa}$.

According to the different models and specifications of transformer, heating stage and intermediate pressure lower stage can be repeated cyclically for many times.

(4) High Vacuum Stage. The high vacuum stage is needed when the moisture content in insulation material is very little, less than $10 \%$ of the total initial moisture content. This part of moisture is dispersed in insulation material, especially in thicker insulation parts, and is difficult to be drawn out. The method of increasing temperature is unavailable to accelerate the moisture evaporation because the temperature of transformer is as high as the allowable temperature of insulation material at this moment. Therefore, the method of reducing the external pressure is adopted. The transformer tank is deeply evacuated in order to make the solvent and moisture further vaporize until the drying standard is reached.

(5) Oil Filling Stage. After the moisture and solvent in insulation material are drawn out, the space occupied by original moisture and solvent should be immediately filled with electric insulation oil in order to prevent absorbing moisture again in space with larger humidity. Under the vacuum condition, transformer oil is poured into the tank to fully saturate insulation parts.

The MVPD equipment is always automatically recording and monitoring the important technical parameters of drying process in the whole working process, such as the temperature of transformer body, the gas pressure inside the tank, and the total water yield. According to the process parameters, operators can make decisions about the subsequent process.

\section{Heat and Mass Transfer Model of MVPD System}

3.1. System Simplification, Variable Definition, and Basic Assumption. In order to more clearly show the internal structure relationship and material flow direction in the MVPD

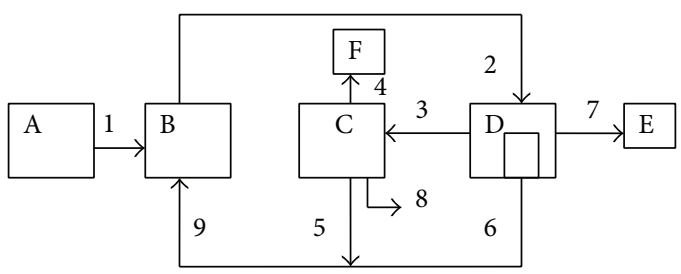

FIGURE 3: Simplified calculation process model of MVPD system.

equipment, the system structure is simplified as a model of calculation flow chart shown in Figure 3. The specific meanings of each symbol in this figure, the process units expressed with alphabet letters, and the process flow expressed with numbers have been shown in Table 1 .

Before setting up a mathematical model, the symbols and units of required physical quantities are defined and shown in Table 2 while the first and second subscript of each physical quantity are defined and shown in Table 3.

To simplify calculation, the fundamental assumptions for system are made as follows.

(1) Ignore the heat loss to surrounding environment in the whole process, because this part of heat does not take part in the internal heat and mass transfer of MVPD system.

(2) Neglect the latent heat of moisture content in insulation material, because it is much smaller than the total heat exchange.

(3) Assume that the temperature of the whole transformer body changes evenly; that is, a lumped parameter model is adopted for the transformer tank.

(4) Assume that the flow of solvent vapor and water vapor in pipes is a steady Poiseuille's laminar flow.

\subsection{Unit Model of MVPD Equipment}

3.2.1. Unit Model of Evaporator. The substance composition in evaporator $B$ is only solvent, which is in two phase states, liquid solvent and solvent vapor. The liquid solvent flows into the evaporator through the logistics pipelines 1 and 9, absorbs heat provided by the heater, and evaporates to become the solvent vapor. The solvent vapor flows out of the evaporator through the logistics pipeline 2. The mathematical model reflecting the unit process includes the following equations. 
TABLE 2: Definition of physical quantity symbols and units.

\begin{tabular}{lc}
\hline$M$ & Mass $(\mathrm{kg})$ \\
$Q$ & Heat $(\mathrm{J})$ \\
$T$ & Temperature $(\mathrm{K})$ \\
$q$ & Heat flow $(\mathrm{w})$ \\
$m$ & Mass flow $(\mathrm{kg} / \mathrm{s})$ \\
$g$ & Phase change rate $(\mathrm{kg} / \mathrm{s})$ \\
$h$ & Phase change latent heat $(\mathrm{J} / \mathrm{kg})$ \\
$\tau$ & Time $(\mathrm{s})$ \\
$L$ & Length $(\mathrm{m})$ \\
$r$ & Radius $(\mathrm{m})$ \\
$S$ & Area $\left(\mathrm{m}^{2}\right)$ \\
$c$ & Ppecific heat capacity $(\mathrm{J} / \mathrm{kg} \cdot \mathrm{K})$ \\
$p$ & Power $(\mathrm{W})$ \\
$P$ & Pressure $(\mathrm{Pa})$ \\
$\rho$ & Density $\left(\mathrm{kg} / \mathrm{m}^{3}\right)$ \\
$\eta$ & Viscosity coefficient $(\mathrm{Pa} \cdot \mathrm{s})$ \\
$\partial$ & Boiling heat transfer coefficient $\left(\mathrm{w} / \mathrm{m}^{2} \cdot \mathrm{K} \cdot \mathrm{Pa}\right)$ \\
$\beta$ & Condensation surface coefficient $\left(\mathrm{w} / \mathrm{m}^{2} \cdot \mathrm{K} \cdot \mathrm{Pa}\right)$ \\
$\lambda$ & Convective heat transfer coefficient $(\mathrm{w} / \mathrm{m} \cdot \mathrm{K})$ \\
$\mu$ & Molar mass $(\mathrm{kg} / \mathrm{mol})$ \\
$C$ & Constant \\
$k$ & Coefficient \\
\hline
\end{tabular}

TABLE 3: Definition of the first and second subscripts of each physical quantity.

\begin{tabular}{lcc}
\hline \multirow{3}{*}{ The first subscript } & A B C D E F & Process unit label \\
& 123456789 & Process logistics label \\
& I II III IV V & Time phase \\
\hline & $S$ & Liquid solvent \\
& $O$ & Solvent vapor \\
The second subscript & $W$ & Liquid water \\
& $A$ & Water vapor \\
& $j$ & Air \\
& $i$ & Insulation materials \\
& $b$ & Transformer tank \\
& $c$ & Copper wire \\
\hline
\end{tabular}

The mass of liquid solvent in $B$ is $M_{B S}$ :

$$
\frac{d M_{B S}}{d \tau}=m_{1 S}+m_{9 S}-g_{B O} .
$$

The mass of solvent vapor in $B$ is $M_{B O}$ :

$$
\frac{d M_{B O}}{d \tau}=g_{B O}-m_{2 O}
$$

Phase change rate is $g_{B O}$ :

$$
\begin{aligned}
g_{B O} & =\partial_{B S} \cdot \sqrt{\frac{\mu_{B S}}{2 \pi R}} \cdot\left(\frac{P_{B S}}{\sqrt{T_{B S}}}-\frac{P_{B O}}{\sqrt{T_{B O}}}\right) \cdot S_{B} \\
& =k_{B S} \cdot\left(\frac{P_{B S}}{\sqrt{T_{B S}}}-\frac{P_{B O}}{\sqrt{T_{B O}}}\right),
\end{aligned}
$$

where $k_{B S}=\partial_{B S} \cdot \sqrt{\mu_{B S} / 2 \pi R} \cdot S_{B}$.
The temperature of liquid solvent is $T_{B S}$ :

$$
c_{O} \cdot M_{B S} \frac{d T_{B S}}{d \tau}=p_{B}-g_{B O} \cdot h_{B S} .
$$

The temperature of solvent vapor is $T_{B O}$ :

$$
T_{B O}=T_{B S} .
$$

According to the test data provided by a manufacture factory, the saturated vapor pressure of liquid solvent can be computed by the following equation:

$$
\lg P_{B S}=10.1-\frac{2153.6}{T_{B S}-34.3} .
$$

The pressure of solvent vapor is $P_{B O}$ and it may be computed by the perfect gas state equation under low pressure:

$$
P_{B O}=\frac{M_{B O} R T_{B O}}{V_{B} \mu_{B O}} .
$$

3.2.2. Unit Model of Transformer. The substances composition concerned in the heat and mass transfer process in the transformer $D$ consists of solvent, water, and air. There are two phase states including liquid state and vapor state for solvent and water. The solvent vapor flows into the transformer tank through the logistics pipeline 2 and then condenses and turns into liquid solvent. The heat released by phase change will heat the transformer tank, iron cores, copper wires, and insulation material. The mass conversion and heat exchange in this unit can be expressed by the following mathematical equations.

(1) Air. The mass of air is $M_{D A}$ :

$$
\frac{d M_{D A}}{d \tau}=m_{A}-m_{3 A},
$$

where $m_{A}$ is the amount of leakage air.

The air temperature is $T_{D A}$ and it is equal to the temperature of solvent vapor $T_{D O}$ in unit $D$ :

$$
T_{D A}=T_{D O}
$$

The partial pressure of air in the transformer $D$ is $P_{D A}$ :

$$
P_{D A}=\frac{M_{D A} R T_{D A}}{V_{D} \mu_{D A}} .
$$

(2) Water. The water in the transformer $D$ includes the liquid absorbed water existing in the insulation material and the water vapor evaporating into the space. About the water vapor see the following.

The mass of water vapor is $M_{D V}$ :

$$
\frac{d M_{D V}}{d \tau}=g_{D V}-m_{3 V}
$$

The temperature of water vapor is $T_{D V}$ and it is equal to the temperature of solvent vapor $T_{D O}$ :

$$
T_{D V}=T_{D O} .
$$


The partial pressure of water vapor is $P_{D V}$ :

$$
P_{D V}=\frac{M_{D V} R T_{D V}}{V_{D} \mu_{D V}} .
$$

About the liquid water see the following.

The mass of liquid water is $M_{D W}$ :

$$
\frac{d M_{D W}}{d \tau}=-g_{D V}
$$

The temperature of liquid water is $T_{D W}$ and it is equal to the temperature of transformer body $T_{D}$ :

$$
T_{D W}=T_{D}
$$

The saturation pressure of liquid water is $P_{D W}$ given by the following equation $[15,16]$ :

$$
\lg P_{D W}=10.07406-\frac{1657.46}{T_{D W}-45.98} .
$$

The evaporation phase change rate of liquid water is $g_{D V}$ :

$$
\begin{aligned}
g_{D V} & =\partial_{D W} \cdot \sqrt{\frac{\mu_{D W}}{2 \pi R}} \cdot\left(\frac{P_{D W}}{\sqrt{T_{D}}}-\frac{P_{D V}}{\sqrt{T_{D V}}}\right) \cdot S_{D} \\
& =k_{D W} \cdot\left(\frac{P_{D W}}{\sqrt{T_{D}}}-\frac{P_{D V}}{\sqrt{T_{D V}}}\right),
\end{aligned}
$$

where $k_{D W}=\partial_{D W} \cdot \sqrt{\mu_{D W} / 2 \pi R} \cdot S_{D}$.

(3) Solvent. The solvent includes the solvent vapor entering through the logistics pipeline 2 and the liquid solvent produced by condensation phase change. Most of the liquid solvent flows out through the logistics pipeline 6 , and the rest retains on the wall surface of transformer tank and in the insulation material.

About the solvent vapor see the following.

The mass of solvent vapor is $M_{D O}$ :

$$
\frac{d M_{D O}}{d \tau}=m_{2 O}-g_{D O}-m_{3 O}
$$

The temperature of solvent vapor is $T_{D O}$ :

$$
T_{D O}=T_{B O}-\Delta T,
$$

where $\Delta T$ is the temperature loss of pipeline $2, \Delta T=3 \mathrm{~K}$.

The pressure of solvent vapor is $P_{D O}$ :

$$
P_{D O}=\frac{M_{D O} R T_{D O}}{V_{D} \mu_{D O}} .
$$

The condense-phase-change rate of solvent vapor is $g_{D O}$ :

$$
\begin{aligned}
g_{D O} & =\beta_{D O} \cdot \sqrt{\frac{\mu_{D O}}{2 \pi R}} \cdot\left(\frac{P_{D O}}{\sqrt{T_{D O}}}-\frac{P_{D S}}{\sqrt{T_{D S}}}\right) \cdot S_{D} \\
& =k_{D O} \cdot \beta_{D O} \cdot\left(\frac{P_{D O}}{\sqrt{T_{D O}}}-\frac{P_{D S}}{\sqrt{T_{D S}}}\right),
\end{aligned}
$$

where $k_{D O}=\sqrt{\mu_{D O} / 2 \pi R} \cdot S_{D}$.
The condensation heat transfer coefficient of solvent vapor in the tank is $\beta_{D O}$ :

$$
\beta_{D O}=k_{D}\left(C_{D S}^{2}-M_{D S}^{2}\right) .
$$

$\beta_{D O}$ is related to the mass of condensed liquid solvent. The condensation heat transfer coefficient is decreasing with the mass increasing of condensed liquid solvent. The maximum mass of condensed liquid solvent in the tank is defined as $C_{D S}$. When $M_{D S}=C_{D S}$, then $\beta_{D O}=0$, which means that the solvent vapor will no longer condense and the solvent vapor entering the tank is mainly taken out by the leakage pump through the logistics pipeline 3. In this case, the intermediate pressure lower stage should be entered.

About the liquid solvent see the following.

The mass of liquid solvent is $M_{D S}$ :

$$
\frac{d M_{D S}}{d \tau}=g_{D O}-m_{6 S}
$$

The temperature of liquid solvent is $T_{D S}$ :

$$
T_{D S}=T_{D}
$$

The saturation pressure of liquid solvent is $P_{D S}$ :

$$
\lg P_{D S}=10.1-\frac{2153.6}{T_{D S}-34.3} .
$$

(4) Transformer Body. The transformer body includes iron cores, copper wires, and insulation materials of transformer core and transformer tank. The temperature of transformer body is $T_{D}$ :

$$
\begin{aligned}
& \left(c_{D j} M_{D j}+c_{D c} M_{D c}+c_{D i} M_{D i}+c_{D b} M_{D b}\right) \frac{d T_{D}}{d \tau} \\
& =g_{D O} \cdot h_{D S}-m_{6 S} \cdot h_{6 S}+\lambda_{D O} \cdot S_{D} \cdot\left(T_{D O}-T_{D S}\right) .
\end{aligned}
$$

\subsection{Process Logistics Model of the MVPD Equipment}

(1) Logistics 2. There is only the solvent vapor in the logistics pipeline 2 and the flow from evaporator $B$ towards transformer $D$ is in accordance with the Poiseuille flow. The flux of logistics 2 depends on the pressure difference between the solvent vapor pressure in the evaporator and the total pressure of mixed gas in the transformer, as well as the structure of pipeline 2 .

The volume flow of solvent vapor is $Q_{2}$ :

$$
Q_{2 O}=\frac{\pi r_{2}^{4}}{16 \eta_{2} L_{2}} \cdot\left(P_{B O}^{2}-P_{D T}^{2}\right) .
$$

The mass flow of solvent vapor is $m_{2 O}$ :

$$
\begin{aligned}
m_{2 O} & =\rho_{2 O} \cdot Q_{2 O} \\
& =\frac{P_{B O} \mu_{B O}}{R T_{B O}} \cdot \frac{\pi r_{2}^{4}}{16 \eta_{2} L_{2}} \cdot\left(P_{B O}^{2}-P_{D T}^{2}\right) \\
& =k_{B O} \cdot \frac{P_{B O}}{T_{B O}} \cdot\left(P_{B O}^{2}-P_{D T}^{2}\right),
\end{aligned}
$$

where $k_{B O}=\left(\mu_{B O} / R\right) \cdot\left(\pi r_{2}^{4} / 16 \eta_{2} L_{2}\right)$. 
(2) Logistics 3. The substance composition in logistics 3 includes solvent vapor, water vapor, and air. The flow of mixture from transformer $D$ towards condenser $C$ is in accordance with Poiseuille flow. The flux of mixed gas depends on the pressure difference between the total pressure in condenser $C$ and the total pressure in the transformer, as well as the structure of pipeline 3. The volume flow ratio of each gas is proportional to the partial pressure ratio of this gas in transformer $D$. The parameters $m_{3 A}, m_{3 V}$, and $m_{3 O}$ in (8), (11), and (18) can be calculated through the following equation.

The total volume flow of mixed gas is $Q_{3 T}$ :

$$
Q_{3 T}=\frac{\pi r_{3}^{4}}{16 \eta_{D T} L_{3}} \cdot\left(P_{D T}^{2}-P_{C}^{2}\right),
$$

where the total pressure of mixed gas is equal to the sum of each gas partial pressure; namely,

$$
P_{D T}=P_{D O}+P_{D A}+P_{D V}
$$

The equivalent viscosity coefficient of mixed gas depends on the viscosity coefficient of each gas and the proportion of mixed gas; namely,

$$
\begin{aligned}
\eta_{D T}= & \eta_{D A} \cdot \frac{\rho_{D A}}{\rho_{D A}+\rho_{D O}+\rho_{D V}} \\
& +\eta_{D O} \cdot \frac{\rho_{D O}}{\rho_{D A}+\rho_{D O}+\rho_{D V}}+\eta_{D V} \cdot \frac{\rho_{D V}}{\rho_{D A}+\rho_{D O}+\rho_{D V}}
\end{aligned}
$$

The volume flow of air in mixed gas is

$$
Q_{3 A}=\frac{P_{D A}}{P_{D T}} \cdot Q_{3 T} .
$$

The volume flow of water steam in mixed gas is

$$
Q_{3 V}=\frac{P_{D V}}{P_{D T}} \cdot Q_{3 T} \cdot
$$

The volume flow of solvent vapor in mixed gas is

$$
Q_{3 O}=\frac{P_{D O}}{P_{D T}} \cdot Q_{3 T} .
$$

If the volume flow is converted into the mass flow, the mass flow of air in mixed gas is

$$
m_{3 A}=\frac{P_{D A} \mu_{D A}}{R T_{D}} \cdot Q_{3 A} .
$$

The mass flow of water steam in mixed gas is

$$
m_{3 V}=\frac{P_{D V} \mu_{D V}}{R T_{D}} \cdot Q_{3 V} .
$$

The mass flow of solvent vapor in mixed gas is

$$
m_{3 O}=\frac{P_{D O} \mu_{D O}}{R T_{D}} \cdot Q_{3 O} .
$$

(3) Other Logistics. The mass flow of condensed solvent liquid in logistics 6 is directly related to the phase change rate of solvent vapor in transformer $D$. Consider the following:

$$
m_{6 S}=k_{6 S} \cdot g_{D S}
$$

The mass flow of condensed solvent liquid in logistics 5 is equal to the mass flow of solvent vapor in flow 3:

$$
m_{5 S}=m_{3 O}
$$

The mass flow of liquid solvent in logistics 9 is equal to the sum of logistics 5 and logistics 6 :

$$
m_{9 S}=m_{6 S}+m_{5 S}
$$

\section{Simulation and Discussion}

Based on the mathematical model given above, we can carry out the numerical simulation of MVPD process. Under the limit of thesis space, the model established above and the following simulation will focus on the heat and mass transformation and transfer process of various working medium in evaporator $B$ and vacuum chamber $D$. Much attention is paid to the variations of the transformer body temperature, the pressure in the tank, and the water yield while the process parameters changes of other auxiliary process units and flows are not concerned.

4.1. Simulation Calculation Flow Chart. The simulation is in accordance with time sequence and time is taken as the independent variable; simulation process starts at a time point when all necessary technological parameters are given as the initial condition. The recurrence method is used to extrapolate calculation value of every variable at the next moment in turn (time step is 1 second). Every simulation cycle finishes a whole MVPD process (about dozens of hours). In the process of simulation, the end criterion conditions of preparation stage, heating stage, intermediate pressure lower stage, and high vacuum stage are set. As simulation parameters reach the end criterion conditions, simulation program will shift to the next stage automatically. The simulation calculation flow chart of MVPD process is shown in Figure 4.

4.2. Calculation Example. A simulation example is given below. This example comes from an actual field drying process of a transformer. The voltage insulation class of this large power transformer is $500 \mathrm{kV}$ and the capacity of it is 750 MVA. The MVPD 8.2 equipment is adopted in actual production to perform dry processing.

According to the formulas of heat and mass transfer model of MVPD system, simulation calculation procedure is programmed with MATLAB software. Based on actual process, the whole simulation time of heating stage and intermediate pressure lower stage is set as 66 hours, and time step is 1 second. The structural and physical parameters of both transformer and MVPD equipment used in simulation calculation and the constant parameters in simulation process are listed in Table 4. 
TABLE 4: Parameters used in simulation process.

\begin{tabular}{|c|c|c|c|}
\hline The physical meaning of parameters & Symbol & Value & Unit \\
\hline Voltage class & $U$ & 500 & $\mathrm{kV}$ \\
\hline Transformer capacity & $I$ & 750 & MVA \\
\hline Flow of logistics 1 & $m_{1 s}$ & 1.35 & $\mathrm{~kg} / \mathrm{s}$ \\
\hline Universal gas constant & $R$ & 8.314 & {$[\mathrm{~J} /(\mathrm{mol} \cdot \mathrm{K})]$} \\
\hline Heating power & $p_{B}$ & 200 & $\mathrm{~kW}$ \\
\hline Pipe radius of logistics 2 & $r_{2}$ & 125 & $\mathrm{~mm}$ \\
\hline Pipe radius of logistics 3 & $r_{3}$ & 75 & $\mathrm{~mm}$ \\
\hline Pipe length of logistics 2 & $L_{2}$ & 3000 & $\mathrm{~mm}$ \\
\hline Pipe length of logistics 3 & $L_{3}$ & 5000 & $\mathrm{~mm}$ \\
\hline Gas volume of evaporator & $V_{B}$ & 3.14 & $\mathrm{~m}^{3}$ \\
\hline Gas volume of transformer tank & $V_{D}$ & 392.62 & $\mathrm{~m}^{3}$ \\
\hline Heat transfer area of evaporator & $S_{B}$ & 4 & $\mathrm{~m}^{2}$ \\
\hline Heat transfer area of transformer tank & $S_{D}$ & 15 & $\mathrm{~m}^{2}$ \\
\hline Specific heat capacity of solvent vapor & $c_{O}$ & 2015 & $\mathrm{~J} / \mathrm{kg} \cdot \mathrm{K}$ \\
\hline Specific heat capacity of insulation materials & $c_{D j}$ & 1200 & $\mathrm{~J} / \mathrm{kg} \cdot \mathrm{K}$ \\
\hline Specific heat capacity of copper wire & $c_{D c}$ & 390 & $\mathrm{~J} / \mathrm{kg} \cdot \mathrm{K}$ \\
\hline Specific heat capacity of iron core & $c_{D i}$ & 460 & $\mathrm{~J} / \mathrm{kg} \cdot \mathrm{K}$ \\
\hline Specific heat capacity of tank & $c_{D b}$ & 470 & $\mathrm{~J} / \mathrm{kg} \cdot \mathrm{K}$ \\
\hline Mass of insulation materials & $M_{D j}$ & 22000 & $\mathrm{~kg}$ \\
\hline Mass of copper wire & $M_{D c}$ & 30000 & $\mathrm{~kg}$ \\
\hline Mass of iron core & $M_{D i}$ & 180000 & $\mathrm{~kg}$ \\
\hline Mass of box & $M_{D b}$ & 100000 & $\mathrm{~kg}$ \\
\hline Mass density of air & $\rho_{D A}$ & $0.946\left(\right.$ at $\left.130^{\circ} \mathrm{C}\right)$ & $\mathrm{kg} / \mathrm{m}^{3}$ \\
\hline Mass density of solvent & $\rho_{D O}$ & 810 & $\mathrm{~kg} / \mathrm{m}^{3}$ \\
\hline Mass density of water & $\rho_{D V}$ & 958.4 & $\mathrm{~kg} / \mathrm{m}^{3}$ \\
\hline Molar mass of air & $\mu_{D A}$ & 29 & $\mathrm{~g} / \mathrm{mol}$ \\
\hline Molar mass of water steam & $\mu_{D v}$ & 18 & $\mathrm{~g} / \mathrm{mol}$ \\
\hline Molar mass of water & $\mu_{D W}$ & 18 & $\mathrm{~g} / \mathrm{mol}$ \\
\hline Molar mass of solvent vapor & $\mu_{D O}$ & 128 & $\mathrm{~g} / \mathrm{mol}$ \\
\hline Molar mass of solvent liquid & $\mu_{D S}$ & 128 & $\mathrm{~g} / \mathrm{mol}$ \\
\hline Solvent enthalpy in the evaporator & $h_{B s}$ & 180000 & $\mathrm{~J} / \mathrm{kg}$ \\
\hline Solvent enthalpy in logistics 6 & $h_{6 s}$ & 40000 & $\mathrm{~J} / \mathrm{kg}$ \\
\hline Solvent enthalpy in transformer tank & $h_{D s}$ & 180000 & $\mathrm{~J} / \mathrm{kg}$ \\
\hline Viscosity coefficient of logistics 2 & $\eta_{2}$ & $7.52 \times 10^{5}$ & $\mathrm{~Pa} \cdot \mathrm{s}$ \\
\hline Viscosity coefficient of air & $\eta_{D A}$ & $1.79 \times 10^{-5}$ & $\mathrm{~Pa} \cdot \mathrm{s}$ \\
\hline Viscosity coefficient of solvent vapor & $\eta_{D O}$ & $7.52 \times 10^{5}$ & $\mathrm{~Pa} \cdot \mathrm{s}$ \\
\hline Viscosity coefficient of water steam & $\eta_{D V}$ & $2.838 \times 10^{8}$ & $\mathrm{~Pa} \cdot \mathrm{s}$ \\
\hline Heat transfer coefficient of water & $\partial_{D W}$ & 0.284 & $\mathrm{~W} / \mathrm{m}^{2} \cdot \mathrm{Pa}$ \\
\hline Heat transfer coefficient of solvent & $\partial_{B S}$ & 0.5 & $\mathrm{~W} / \mathrm{m}^{2} \cdot \mathrm{Pa}$ \\
\hline Maximum mass of condensed solvent in tank & $C_{D S}$ & 100 & $\mathrm{~kg}$ \\
\hline Leakage pump pressure & $P_{C}$ & $\begin{array}{l}4500 \text { (heating) } \\
2500 \text { (IPL) }\end{array}$ & $\mathrm{Pa}$ \\
\hline
\end{tabular}




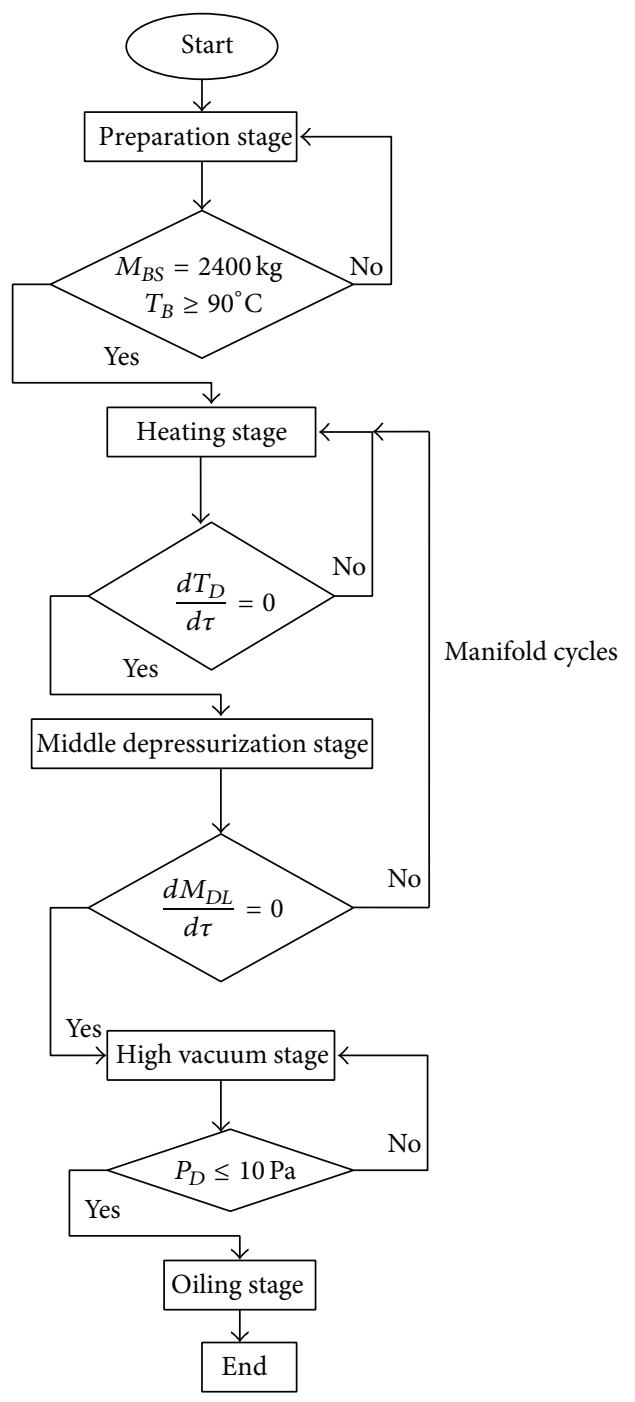

FIGURE 4: The flow chart of calculation program.

4.3. Simulation Results and Discussion. The simulation in this paper focuses on the variation of key process parameters over time such as transformer body temperature, pressure in the tank, and water yield. The change curve of transformer body temperature via time is shown in Figure 5. The change curve of pressure in the tank via time is shown in Figure 6. Total water yield $M_{D L}$ in the operation process via time is the most intuitive index parameter reflecting transformer drying effect. It should be equal to the difference between the initial moisture content $M_{D W 0}$ of insulation materials and the residual moisture content $M_{D W}$.

Namely,

$$
M_{D L}=M_{D W 0}-M_{D W}
$$

The dehydration rate of insulation materials at a certain time point is

$$
\frac{d M_{D L}}{d \tau}=m_{D V}
$$

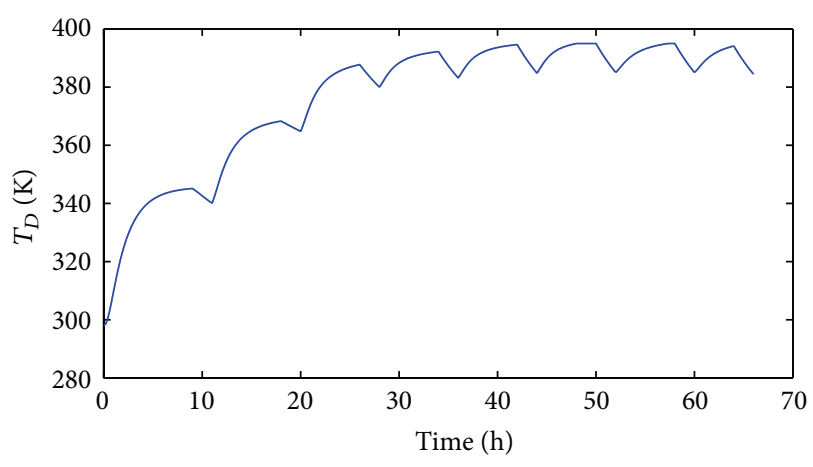

FIGURE 5: Simulation curve of transformer body temperature.

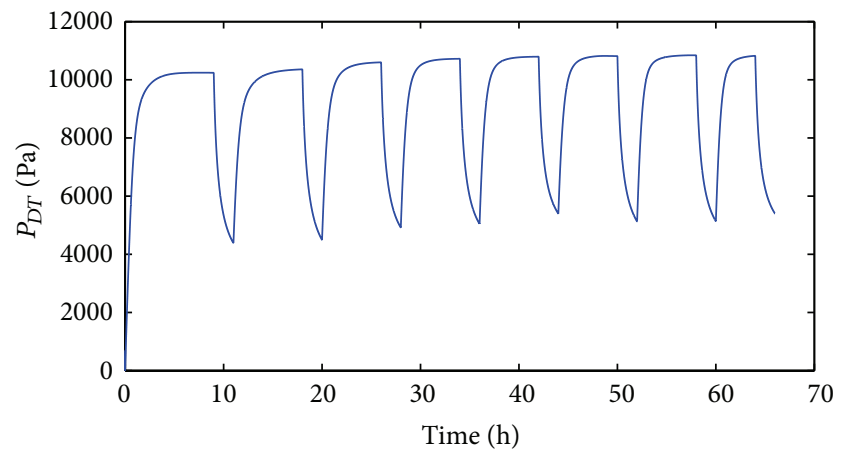

FIGURE 6: Simulation curve of pressure in the tank.

In this simulation result, the simulation curve of total water yield $M_{D L}$ changing over time is shown in Figure 7.

During the actual drying process of $500 \mathrm{kV} / 750 \mathrm{MVA}$ transformer, the MVPD8.2 equipment can record the process parameters of different times by sensors. Summarizing the data and program by VB, finally the actual process curve of MVPD can be got as shown in Figure 8 . Figure 8 has shown, respectively, the change law of key process parameters over time such as temperature value, pressure value, and water yield.

By comparing the simulated curve and measured curve, we can find that the simulation results are quite coincident with the measured values in the aspect of change trend of key parameters. Both transformer body temperature and pressure in the tank increase at heating stage and decrease at intermediate pressure lower stage. Besides, their range ability and change law are also very consistent.

Comparison results show that the mathematical model established and the calculation procedure programmed can correctly reflect the heat and mass transfer law of MVPD process and can predict quantitatively the change trend of key physical parameters [17].

The obvious difference between the simulation results and the practical measured values is as follows. The simulation calculation shows that only 8 times heating stages and 7 times intermediate pressure lower stages are enough for the whole drying process, while 11 times heating stages and 10 times intermediate pressure lower stages were used in the actual operation process. The reason for this difference is that 


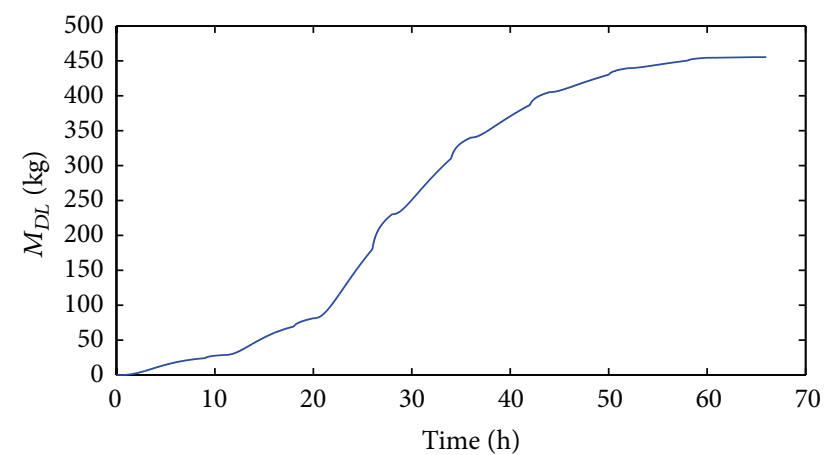

FIGURE 7: Simulation curve of total water yield.

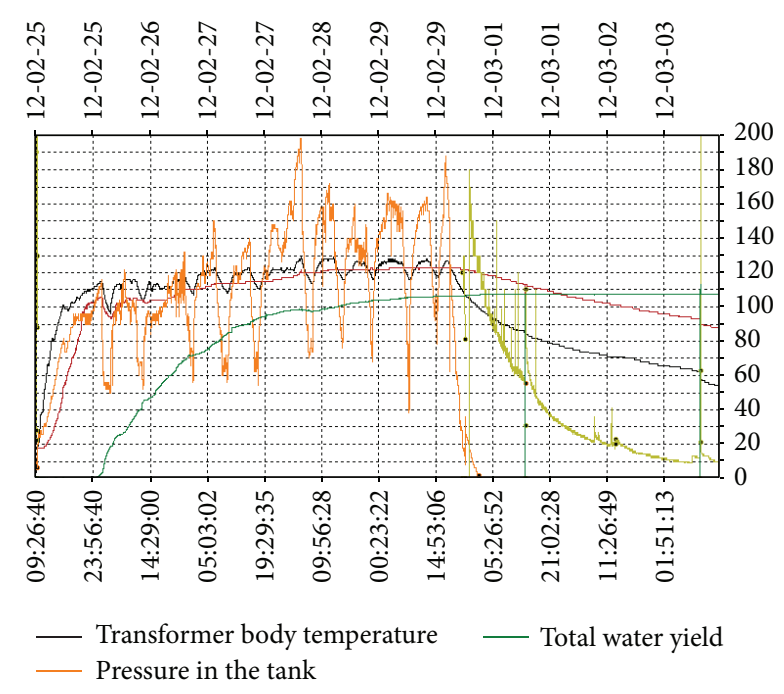

FIgURE 8: Actual craft process curve of MVPD 8.2.

the end criterion conditions and the judgment of every process transformation are different. In the practical operation process, operators usually depend on their practical experience.

In practical MVPD process, when the ascent rate of body temperature is less than a setting value and the pressure in the tank is greater than a setting value, heating stage will be stopped and intermediate pressure lower stage will be started, so saving the process time. However, the end criterion conditions of heating stage set in this program are that the ascent rate of transformer body temperature is zero. Therefore, the times of process transformation in simulated calculation are less.

Based on the mathematical model established and the simulation procedure programmed in this paper, by analyzing the simulation results, some reasonable and useful conclusions can be obtained. The optimal values of some process parameters can be speculated so that the optimization of structure and process parameters of MVPD can be done. For example, the value of heating power $P_{B O}$, which should make sure that evaporator $B$ can produce proper quantity of solvent vapor, can be calculated by the flux of solvent vapor in logistics 2, and the simulation result is shown in Figure 9. This value may direct the power control of the heater in evaporator $B$ as a supplement of the negative feedback control used in practical MVPD equipment.

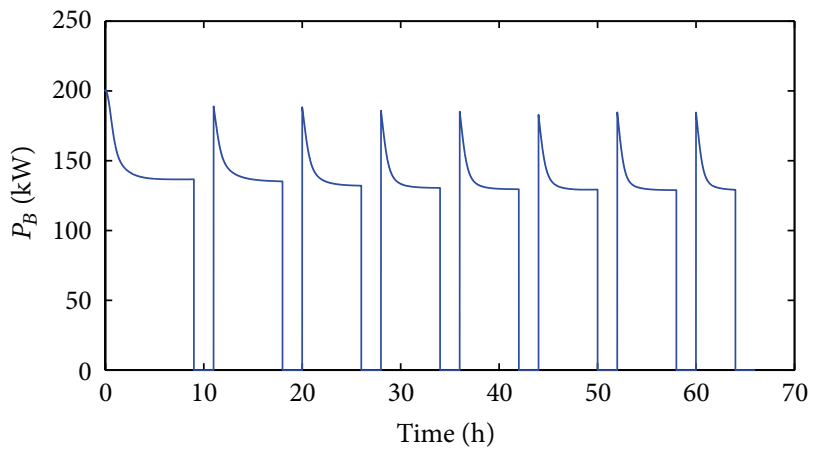

FIGURE 9: Curve of evaporator heat power via time in the drying process.

\section{Conclusion}

On the basis of analyzing in detail the working principle, system composition, and technological process of the MVPD equipment, a simplified process model reflecting the relationship between internal operation units and processes is extracted in this paper. According to the principle of mass and energy conservation, a heat mass transfer mathematical model including 40 mathematical equations is established. This mathematical model represents completely thermodynamics laws of phase change and migration process of solvent, water, and air in technological process, describes in detail the quantitative relationship among important physical quantities such as temperature, pressure, and flux in key equipment units and processes logistics, and reflects the internal mechanism of heat and mass transfer and conversion.

According to this mathematical model and taking an actual drying technological process of a $500 \mathrm{kV} / 750$ MVA transformer as example, the simulation calculation of a complete technological process treatment is finished by programming with MATLAB software. Simulation results give the change law of key process parameters over time such as transformer body temperature, pressure in the tank, and water yield. Comparing the simulation results and the actual record data, it is found that their change trend are very consistent with each other, which verifies the correctness of mathematical model established.

In the future, based on the mathematical model, the influence of structure parameters and operation process parameters of the MVPD equipment on the whole drying process and drying effect can be studied quantitatively. This mathematical model can be applied to provide theoretical basis for the drying technological process control and result prediction and can serve as a reference for relevant researchers in optimizing the equipment structure design and process operation.

\section{Conflict of Interests}

The authors declare that there is no conflict of interests regarding the publication of this paper. 


\section{Acknowledgments}

This research was supported by National Natural Science Foundation of China (Grant nos. 31000665, 51176027, 31371873, and 31300408) and the Fundamental Research Funds for the Central Universities of China (Grant no. N130403001).

\section{References}

[1] Y. Li, The Study of Mobile Oil Vapor Phase Drying Equipment on Oil-Immersed Transformer, North China Electric Power University, 2009 (Chinese).

[2] B. G. Zhang, Q. W. Zhu, and F. Zhang, "Domestic oil vapor phase drying equipment and application," Transformer, no. 10, 1995 (Chinese).

[3] S. W. Ruan, "Oil vapor phase drying principle and process improvement," Process and Technology, vol. 9, 2013 (Chinese).

[4] W. Q. Li, Mechanism and Testing of Moisture Content Measurement of Transformer Pressboard, Dalian University of Technology, 2005, (Chinese).

[5] B. Z. Qiao and S. W. Zhang, "Mobile vacuum vapor phase drying plant and its application," Vacuum, vol. 50, no. 6, pp. 60-67, 2013 (Chinese).

[6] L. R. Liu, "Mobile oil vapor phase drying method and device," North China Electric Power, 2011 (Chinese).

[7] D. F. García, B. García, and J. C. Burgos, "Modeling power transformer field drying processes," Drying Technology, vol. 29, no. 8, pp. 896-909, 2011.

[8] S. D. Foss and L. Savio, "Mathematical and experimental analysis of the field drying of power transformer insulation," IEEE Transactions on Power Delivery, vol. 8, no. 4, pp. 1820-1828, 1993.

[9] W. W. Guidi and H. P. Fullerton, "Mathematical methods for prediction of moisture take-up and removal in large power transformers," in Proceedings of the IEEE Winter Power Meeting, pp. 242-244, 1974.

[10] S. W. Zhang and B. Z. Qiao, "Calculation of heat and mass transfer in the process of transformer vacuum vapor phase drying," Drying Technology and Equipment, vol. 4, no. 3, 2006 (Chinese).

[11] C. H. Xu, S. W. Zhang, and K. Z. Guan, Vacuum Drying, Chemical Industry Press, Beijing, China, 2004, (Chinese).

[12] B. Z. Qiao, Vacuum Vapor Phase Drying and Its Application, Vacuum, 1988 (Chinese).

[13] S. H. Lin, "Prediction of the drying rate of transformer insulation during the dry cycle," Electric Power Systems Research, vol. 23, no. 3, pp. 227-231, 1992.

[14] H. Wildmoser, J. Scheiwiller, and E. J. Windhab, "Impact of disperse microstructure on rheology and quality aspects of ice cream," LWT-Food Science and Technology, vol. 37, no. 8, pp. 881-891, 2004.

[15] M. J. Heathcote, J \& P Transformer Book, pp. 597-763, Elsevier, 2007.

[16] J. H. Keenan and F. G. Keyes, Thermodynamic Properties of Steam, John Wiley \& Sons, New York, NY, USA, 1936.

[17] J. A. Almendros-Ibáñez, Transformer Field Drying Procedures: A Theoretical Analysis, 1978. 


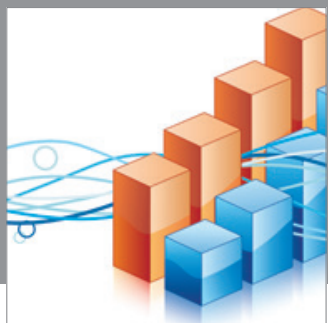

Advances in

Operations Research

mansans

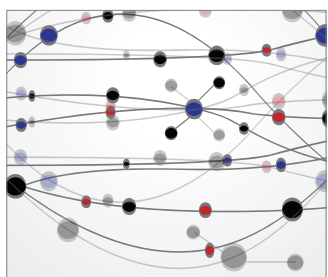

The Scientific World Journal
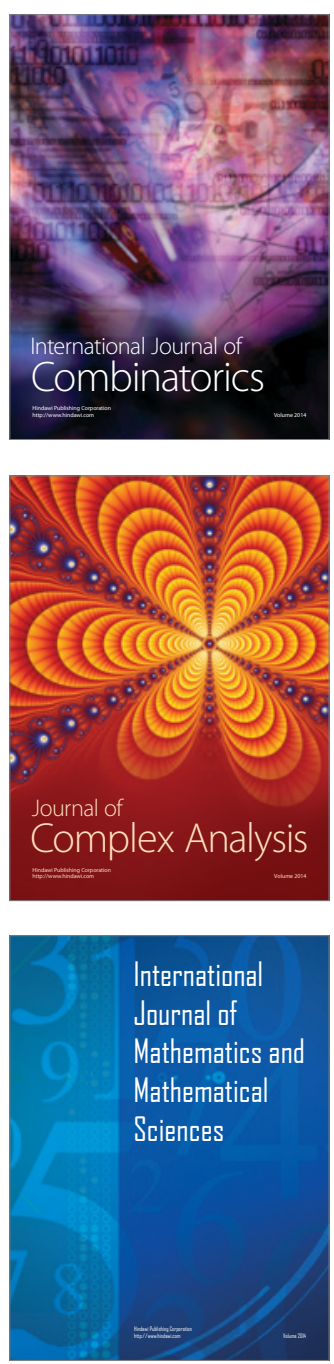
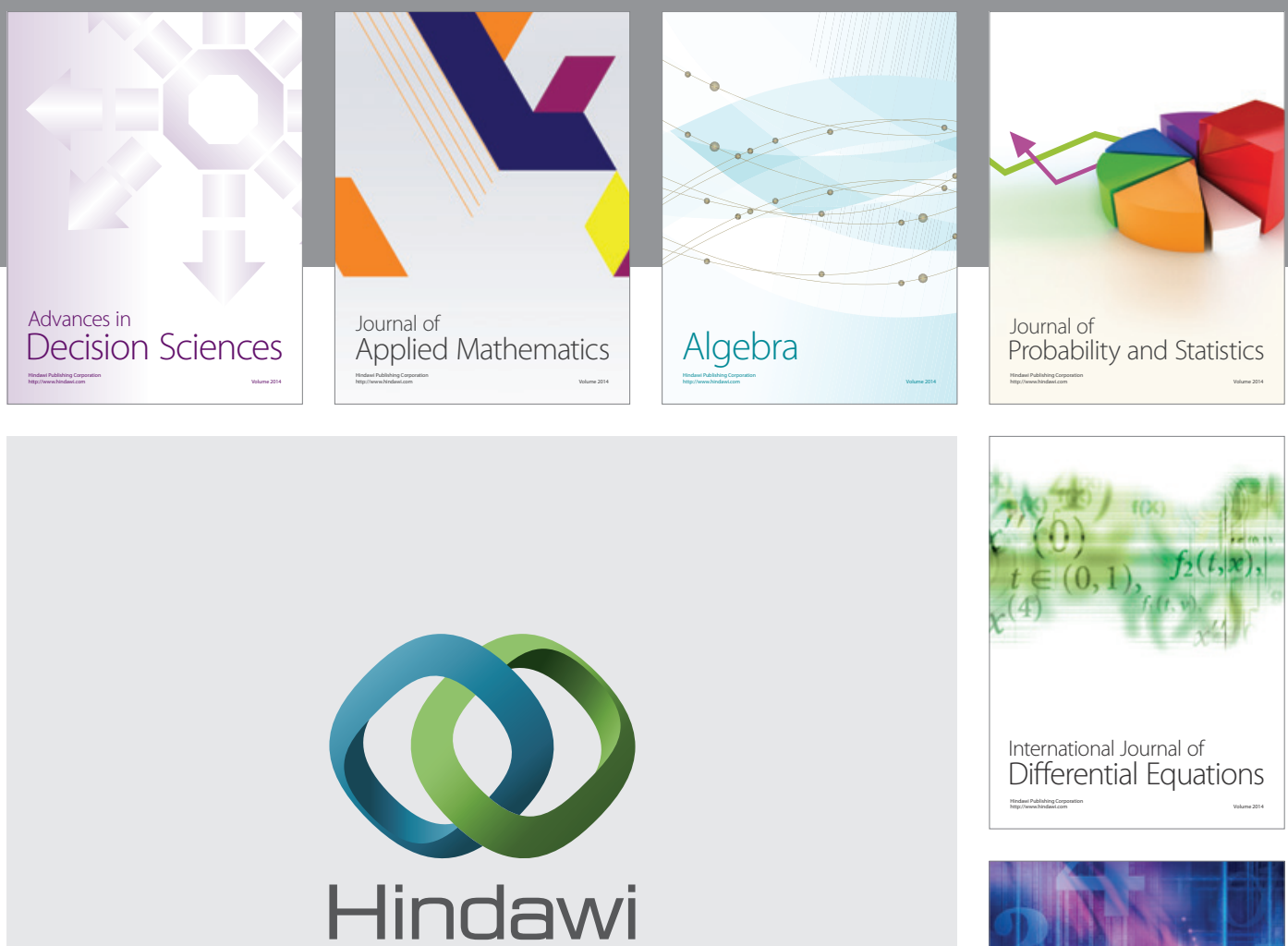

Submit your manuscripts at http://www.hindawi.com
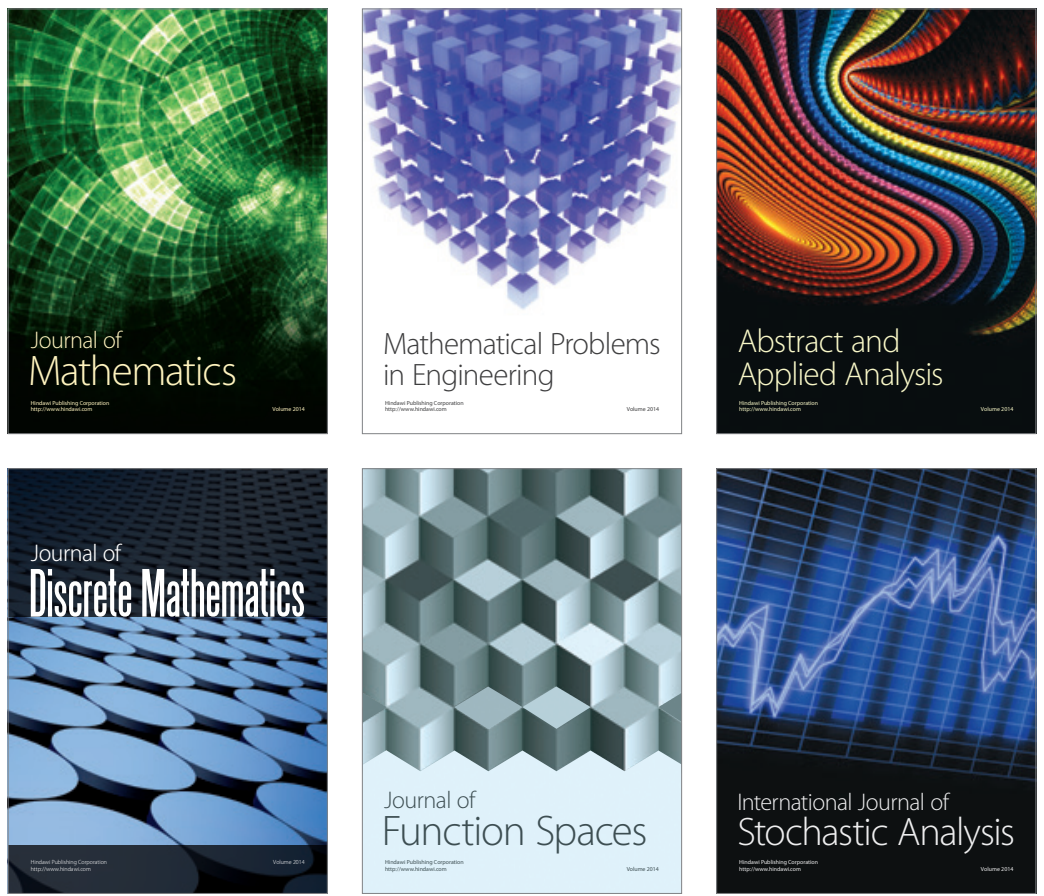

Journal of

Function Spaces

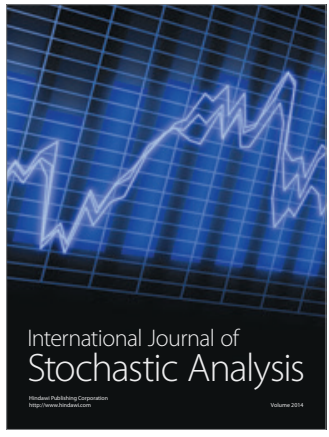

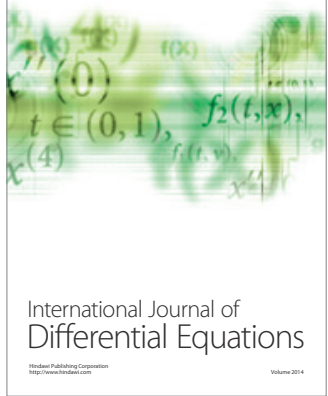
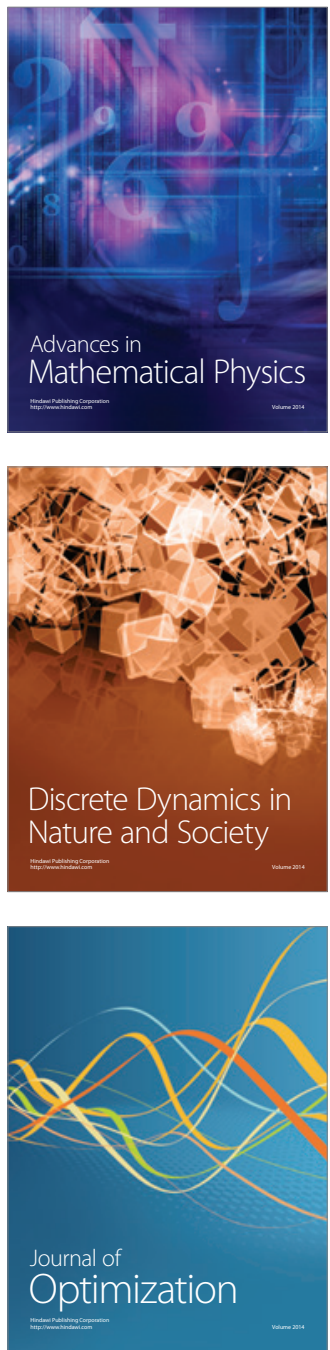